\title{
Device-free Localization of Multiple Targets
}

\author{
Monica Nicoli ${ }^{1}$, Vittorio Rampa ${ }^{2}$, Stefano Savazzi ${ }^{2}$, Silvia Schiaroli ${ }^{1}$ \\ ${ }^{1}$ Dipartimento di Elettronica, Informazione e Bioingegneria (DEIB), Politecnico di Milano \\ ${ }^{2}$ National Research Council of Italy (CNR), IEIIT institute, Milano \\ E-mail: monica.nicoli@polimi.it, vittorio.rampa@ieiit.cnr.it, stefano.savazzi@ieiit.cnr.it
}

\begin{abstract}
In this paper, we consider the problem of multitarget device-free localization with special focus on modeling and inference. The motion of multiple targets inside the area covered by a wireless network leaves a characteristic footprint on the radio-frequency ( $R F)$ field, and in turn affects both the average attenuation and the fluctuation of the received signal strength (RSS). A diffraction-based model is developed to describe the impact of multiple targets on the RSS field, i.e. the multi-bodyinduced shadowing. As a relevant case study, the model is tailored to predict the effects of two co-located targets on the RF signals. Three novel algorithms are proposed for on-line localization, exploiting both the average and the deviation of the body-induced RSS perturbation. The proposed techniques are compared and some preliminary results, based on experimental data collected in a representative indoor environment, are presented.
\end{abstract}

\section{INTRODUCTION}

Recent research has shown that electromagnetic (EM) fields used for data transmission can be exploited as powerful sensing tools for device-free environmental vision [1]. The first experimental activities date back to [2]-[3] and show that body motion leaves a characteristic footprint on the channel quality information, namely on the received signal strength (RSS), which can be exploited for body localization. Perturbations induced on the radio-frequency (RF) signals are processed to extract an image of the environment that originated the perturbation. Radio tomographic imaging (RTI) has been proposed for localization [4] showing that the combination of multiple links enables accurate human-scale understanding. Recent works [5]-[7] focused on assisted living applications [8], including human activity monitoring, body gesture and motion recognition, breathing detection, crowd density estimation, and device-free localization (DFL) (see review in [1]).

In this paper, we propose novel techniques for multi-target DFL. A few systems have been discussed in the literature for multi-target localization [9]-[12], based on either RSS attenuation or variance measurements. The RTI method [9], based on RSS attenuation, models the multi-target RSS footprint as the superposition of the perturbations induced by each individual target. The localization accuracy can be further improved by simultaneous processing of RSS measurements from multiple RF channels [10]. In [11] a linear relationship between the RSS fluctuations and the number of targets in the area has been derived experimentally. A successive-cancellation algorithm is proposed in [12] to address the problem of detection/tracking of new subjects entering/moving in the monitored area.

Accurate multi-target DFL requires the development of simple but realistic EM models to describe multi-body-induced

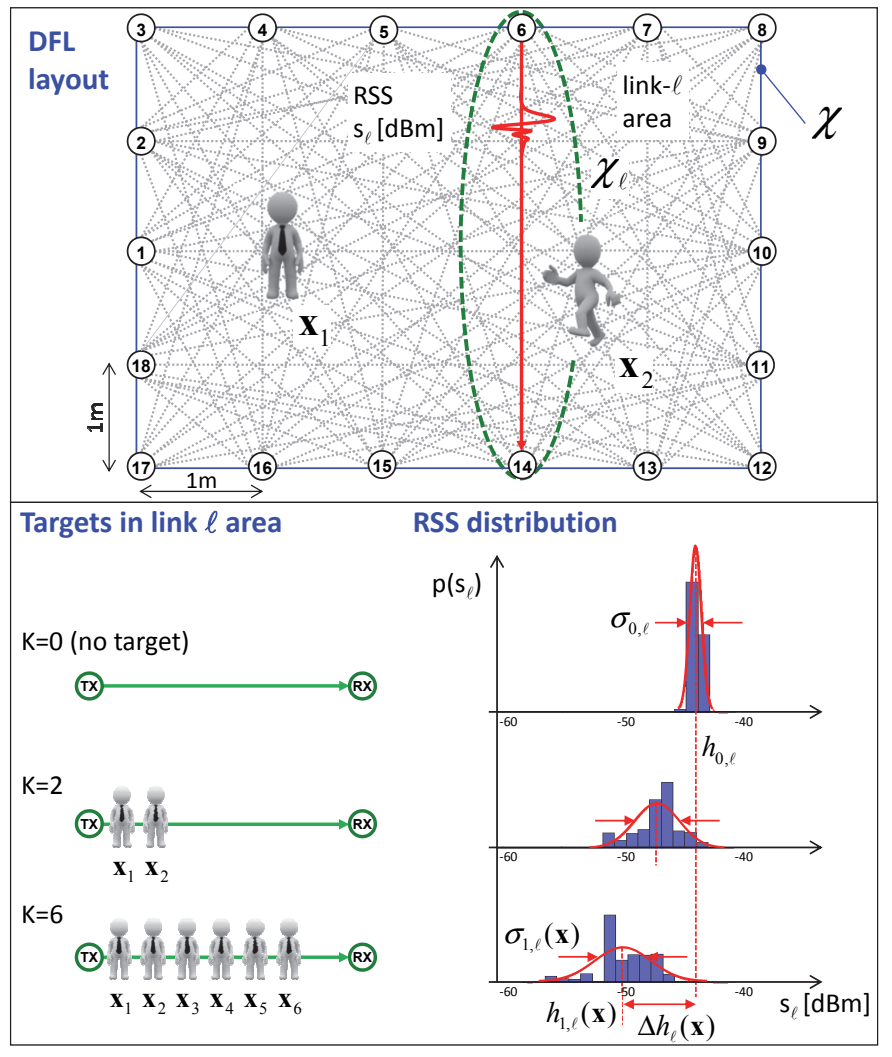

Fig. 1. Multi-target DFL: testing scenario with $N=18$ fully-connected nodes and $K=2$ targets (top); RSS distribution, and related target-induced perturbation, for $K=0,2,6$ targets along the link 6-14 (bottom).

shadowing. Conventional methods based on fingerprinting or ray-tracing are not suited to the multi-user case, due to unfeasible computational burden, while the linear model based on the superposition assumption becomes unrealistic for high people density. Analytical approaches based on propagation modeling in the literature focus on the single-target case only [13]-[15]. Starting from a recent study on single-target humaninduced fading modeling [15], in this paper we propose a new analytical model based on diffraction theory to predict the effects of two co-located targets on both the RSS mean and variance (see Fig. 1). The model can serve as reference for future extension to a larger number of targets in crowded environments and is here validated by extensive measurement data collected by an ad-hoc IEEE 802.15.4 wireless network. In addition, three novel algorithms for multi-target localization are presented, which jointly exploit information on the average 
attenuation and variance of the RSS fluctuation induced by multiple moving targets: joint maximum likelihood (JML), successive cancellation (SC) and a ML-RTI approach. Methods are tested and compared on a real 2-target indoor scenario with network layout as in Fig. 1.

\section{Problem Formulation}

We consider the localization of $K$ human targets at positions $\mathbf{x}_{k}=\left[x_{k, 1}, x_{k, 2}\right]^{\mathrm{T}}, k=1, \ldots, K$, in a two-dimensional (2D) area $\mathcal{X}$ covered by a wireless network of $N$ nodes with $L \leq$ $N(N-1)$ links. The layout selected for experimental testing is shown in Fig. 1 (top), with $K=2$ targets and $N=18$ fully connected nodes. Let $s_{\ell}$ be the RSS in logarithmic scale on link $\ell=1, \ldots, L$, the objective of DFL is the estimation of the $K$ targets' positions $\mathbf{x}=\left[\mathbf{x}_{1}^{\mathrm{T}}, \ldots, \mathbf{x}_{K}^{\mathrm{T}}\right]^{\mathrm{T}}$ from the RSS observations $\mathbf{s}=\left[s_{1} \cdots s_{L}\right]^{\mathrm{T}}$ collected over all the links. According to experimental studies carried out on humaninduced fading for $K=1$ [1][6][15], $s_{\ell}$ can be reasonably modelled as Gaussian distributed ${ }^{1}$ with moments depending on the target location relative to the link, particularly on whether the target location falls or not within the sensitivity area $\mathcal{X}_{\ell}$ of link $\ell$. Extending the model to $K>1$ targets, here the $\operatorname{RSS} s_{\ell}$ is assumed to depend on the number $K_{\ell}(\mathbf{x}) \leq K$ of the targets that are within $\mathcal{X}_{\ell}$ and on their specific locations $\mathbf{x}$ (e.g., $K_{\ell}(\mathbf{x})=1$ in the example of Fig. 1 where only the second target falls in the link- $\ell$ area):

$$
s_{\ell}= \begin{cases}h_{0, \ell}+w_{0, \ell}, & \text { if } K_{\ell}(\mathbf{x})=0 \\ h_{1, \ell}(\mathbf{x})+w_{1, \ell}(\mathbf{x}), & \text { if } K_{\ell}(\mathbf{x})>0\end{cases}
$$

In case of no target in the link area $\left(K_{\ell}(\mathbf{x})=0\right)$, the RSS has deterministic mean $h_{0, \ell}$ that accounts for path-loss and static effects due to fixed obstructing/scattering objects, while the random term $w_{0, \ell} \sim \mathcal{N}\left(0, \sigma_{0, \ell}^{2}\right)$ models the measurement errors as well as small power fluctuations due to variations in the surrounding environment. When one or more targets are within the link area $\left(K_{\ell}(\mathbf{x})>0\right)$, the received power is subject to an increased attenuation due to the obstruction generated by the targets and an amplified fluctuation due to their movements around $\mathbf{x}$ (e.g., turning, change of posture, arm movement, etc.). The mean RSS is thus $h_{1, \ell}(\mathbf{x})=h_{0, \ell}+\Delta h_{\ell}(\mathbf{x}) \leq h_{0, \ell}$, while the random shadowing is $w_{1, \ell}(\mathbf{x}) \sim \mathcal{N}\left(0, \sigma_{1, \ell}^{2}(\mathbf{x})\right)$ with $\sigma_{1, \ell}(\mathbf{x})=\sigma_{0, \ell}+\Delta \sigma_{\ell}(\mathbf{x}) \geq \sigma_{0, \ell}$

Experimental tests on the single-target case [1][6] showed that both the average and standard-deviation perturbations, $\Delta h_{\ell}(\mathbf{x})$ and $\Delta \sigma_{\ell}(\mathbf{x})$, are maximized when the target is obstructing the line-of-sight (LOS), especially if close to the transmitter or the receiver. In the multi-target case, the perturbation is expected to increase with the number of targets. An example is in Fig. 1 (bottom) for a link of length $4 \mathrm{~m}$ with $K=0,2,6$ people along the LOS path with inter-distance $0.5 \mathrm{~m}$. The RSS mean and standard deviation have been calculated by sample averaging over $10 \mathrm{~s}$ while the targets were moving their arms and turning around their positions. The two moments are shown in Fig. 2 for $K$ ranging from 0 to 7 , showing the increasing impact with the number of targets.

\footnotetext{
${ }^{1}$ Although better fits can be provided using other parametric distributions (e.g. Weibull, Nagakami), the approximation is reasonable enough to design the estimation method.
}

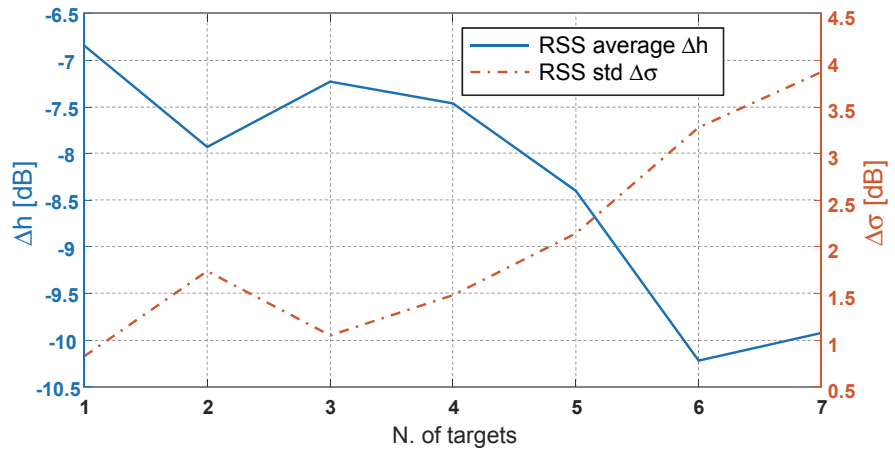

Fig. 2. Mean (blue solid line) and variance (red dashed line) of the RSS fluctuation induced by people moving along the link vs. the number of targets.

From the above examples, both RSS average and variance are expected to provide significant information on the number of targets in the link area and in turn, by combining all links' information, on the target locations in $\mathcal{X}$. We thus propose to jointly exploit RSS mean and standard-deviation observations for multi-target passive localization.

The inference requires the knowledge of the reference parameters $\left\{h_{0, \ell}, \sigma_{0, \ell}\right\}$ (for the empty-link case) as well as the perturbation maps $\left\{\Delta h_{\ell}(\mathbf{x}), \Delta \sigma_{\ell}(\mathbf{x})\right\}$ for all positions $\mathbf{x} \in \mathcal{X}_{\ell}^{K}$ and all links $\ell=1, \ldots, L$. While $\left\{h_{0, \ell}, \sigma_{0, \ell}\right\}$ can be easily pre-calibrated when no target is moving in the area $\mathcal{X}$, evaluation of $\left\{\Delta h_{\ell}(\mathbf{x}), \Delta \sigma_{\ell}(\mathbf{x})\right\}$ is more critical, as it requires extensive fingerprinting campaigns or ray-tracing simulations. The complexity becomes easily cumbersome for $K>1$, even for small number of targets, as the number of configurations to be explored is exponential in $K$. Thereby, analytical modeling is mandatory to simplify the calibration process. In [15], a closed-form analytical model was derived for $K=1$ based on diffraction theory, relating both the RSS average and standard deviation to the target location. The diffraction model was shown to fit reasonably well the shadowing effects of a person or an object placed near the LOS path. In the following section, we propose the extension to $K=2$ targets, accounting for the interactions between the targets. Although not considered in this paper, the proposed approach can be adapted to model the effect of a larger number of targets.

As reference for validation, we also consider the RSS maps evaluated using the conventional fingerprinting approach [6]. As shown in Sect. V, additive perturbation models considered in the literature [10], [4] can be adopted to simplify the problem in exchange for reduced modeling accuracy (due to the linear approximation): the multi-target impact is approximated as the superposition of single-target effects, i.e. $\Delta h_{\ell}(\mathbf{x})=$ $\sum_{k=1}^{K} \Delta h_{\ell}\left(\mathbf{x}_{k}\right)$ and $\Delta \sigma_{\ell}^{2}(\mathbf{x})=\sum_{k=1}^{K} \Delta \sigma_{\ell}^{2}\left(\mathbf{x}_{k}\right)$, with singletarget maps $\Delta h_{\ell}\left(\mathbf{x}_{k}\right)$ and $\Delta \sigma_{\ell}^{2}\left(\mathbf{x}_{k}\right)$ computed assuming one target only, located at position $\mathbf{x}_{k}$. Fingerprinting maps are obtained by collecting RSS samples over each link while a single human target is subsequently positioned over $M$ points $\left\{\mathbf{b}_{m}\right\}_{m=1}^{M}$ of a $2 \mathrm{D}$ grid covering the monitored area.

\section{Modeling of Multi-target RSS Perturbations}

We consider a single-link scenario with $K=2$ threedimensional (3D) objects modeling the human targets, placed 
near the LOS path. The geometrical arrangement in the 3D space $\left(u_{1}, u_{2}, u_{3}\right)$ is shown in Fig. 3, with the transmitter (TX) placed in $(0,0,0)$, the receiver $(\mathrm{RX})$ in $(d, 0,0)$, and the link horizontally laid (along axis $u_{1}$ ) at height $h$ from the floor. The Fresnel's ellipsoid is assumed to have no contact with floor, ceiling, walls or other obstacles except for the two targets: $\min \left(h, d_{w}, d_{c}\right) \gg \sqrt{\lambda d} / 2$, where $\lambda$ is the carrier wavelength, $d_{w}$ and $d_{c}$ are the minimum distances from walls and ceiling. Each 3D object is an homogeneous, perfectly absorbing electromagnetic cylinder, with dimensions as follows: height $2 a_{z}$, elliptical base with semi-axes $a_{y u}>a_{y v}$ for the first obstacle $(k=1)$, and $2 b_{z}$ and $b_{y u}>b_{y v}$ for the second one $(k=2)$. Each object shows a rectangular cross section (i.e., in the plane orthogonal to the LOS path) having height $2 a_{z}$ and traversal semi-size $a_{y}$ for $k=1,2 b_{z}$ and $b_{y}$ for $k=2$. Targets are thus represented as 2D knife-edge obstacles, $S_{k}$, having barycenter $C_{k}=\mathrm{x}_{k}$ located in the horizontal 2D plane containing the LOS path. To model a person that stands in a specific position but might change orientation or posture, we assume that each object can rotate by an angle $\theta_{k}$ around the vertical axis $u_{\theta_{k}}$, such that the traversal semi-size can arbitrarily change in the range $a_{y u} \geq a_{y} \geq a_{y v}$ and $b_{y u} \geq b_{y} \geq b_{y v}$.

According to [15], the electric field $d E$ at the RX, due to all $d S_{k}$ elementary areas of the targets, can be predicted by the forward propagation of the two virtual arrays of Huygens' sources located on the obstacle planes $S_{k}^{\prime}$ but not belonging to the obstacles $S_{k}$ themselves. By the paraxial approximation, neglecting backward/multiple scattering between the obstacles, and ignoring ground, walls and ceilings reflections, we get:

$$
d E=-E_{0} \frac{d}{r_{1 R} r_{21} r_{T 2} \lambda^{2}} e^{-j 2 \pi \frac{r_{T 2}+r_{21}+r_{1 R}-d}{\lambda}} d S_{1} d S_{2}
$$

where $E_{0}$ is the electric field at the RX when no obstacles are present in the link area. By mathematical manipulation of (2), we compute the received electric field $E$ as a function of the link geometry, the position and the size of the objects. By defining the constants $1 / R_{1}^{2}=\left(1 / d_{1}+1 / d_{21}\right) / \lambda, 1 / R_{2}^{2}=$ $\left(1 / d_{2}+1 / d_{21}\right) / \lambda, 1 / R_{21}^{2}=2 /\left(\lambda d_{21}\right), \alpha=R_{1} R_{2} /\left(2 R_{21}^{2}\right)$ and the function $F(\zeta, \xi)=\exp \left(-j \frac{\pi}{2}\left(\zeta^{2}+\xi^{2}-2 \alpha \zeta \xi\right)\right)$, it is:

$$
\frac{E}{E_{0}}=\frac{E_{1}}{E_{0}}+\frac{E_{2}}{E_{0}}+\frac{E_{21}}{E_{0}}-1
$$

where $E_{21} / E_{0}$ models the interaction between the obstacles,

$$
\begin{aligned}
& \frac{E_{21}}{E_{0}}=-\frac{d_{21} d}{4\left(d-d_{1}\right)\left(d-d_{2}\right)} \int_{\frac{y_{1}-a_{y}}{R_{1} / \sqrt{2}}}^{\frac{y_{1}+a_{y}}{R_{1} / \sqrt{2}}} \int_{\frac{y_{2}-b_{y}}{R_{2} / \sqrt{2}}}^{\frac{y_{2}+b_{y}}{R_{2} / \sqrt{2}}} F\left(\zeta_{1}, \zeta_{2}\right) d \zeta_{1} d \zeta_{2} . \\
& \cdot \int_{-\frac{a_{z}}{R_{1} / \sqrt{2}}}^{+\frac{a_{z}}{R_{1} / \sqrt{2}}} \int_{-\frac{b_{z}}{R_{2} / \sqrt{2}}}^{+\frac{b_{z}}{R_{2} / \sqrt{2}}} F\left(\xi_{1}, \xi_{2}\right) d \xi_{1} d \xi_{2},
\end{aligned}
$$

in addition to the superposition of single-target effects $E_{1} / E_{0}$ and $E_{2} / E_{0}$, respectively. Their values can be analytically computed according to [15]. The variables $\zeta_{1}, \zeta_{2}, \xi_{1}, \xi_{2}$ are obtained by using the following substitution rules: $\zeta_{1}=$ $\sqrt{2} u_{2}^{(1)} / R_{1}, \xi_{1}=\sqrt{2} u_{3}^{(1)} / R_{2}, \zeta_{2}=\sqrt{2} u_{2}^{(2)} / R_{1}$ and $\xi_{2}=$ $\sqrt{2} u_{3}^{(2)} / R_{2}$ being $u_{2}^{(k)}, u_{3}^{(k)}$ the generic Cartesian coordinates defined over the $k$-th 2-D obstacle $S_{k}$ and centered on the LOS path. According to (1), it is $\Delta h_{\ell}(\mathbf{x})=E_{\theta}\left[10 \log _{10}\left|E / E_{0}\right|^{2}\right]+$ $\Delta h_{C}$ and $\Delta \sigma_{\ell}(\mathbf{x})=\operatorname{Std}_{\theta}\left[10 \log _{10}\left|E / E_{0}\right|^{2}\right]+\Delta \sigma_{C}$ where

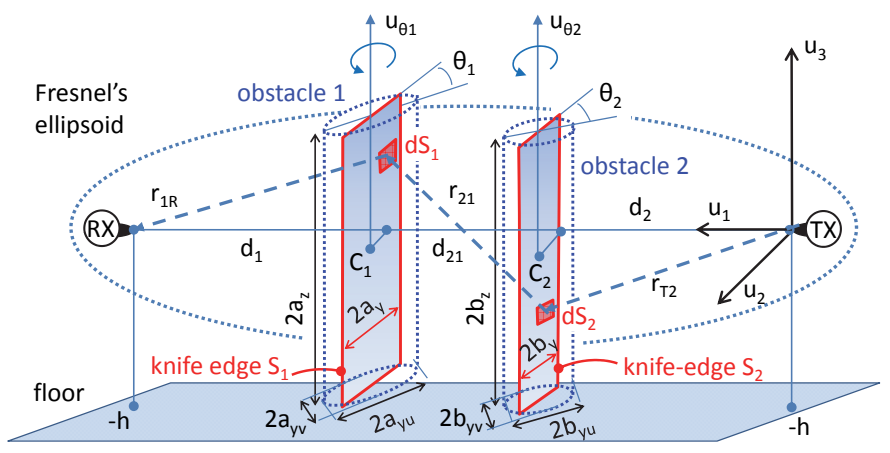

Fig. 3. Geometry of the dual-target scenario for a single link.

$E_{\theta}[\cdot]$ and $\operatorname{Std}_{\theta}[\cdot]$ are the expectation and standard deviation operators with respect to $\theta=\left[\theta_{1}, \theta_{2}\right]$, respectively. $\Delta h_{C}$ and $\Delta \sigma_{C}$ include the multipath effects that are assumed constant in the link area. It is apparent that: $i$ ) being $E_{k i}=0$ and $E_{k}=E_{0}$ if the $k$-th the targets is absent (i.e., only the $i$-th target is present, with $i \neq k$ ), then (3) reduces to the single-target case $E=E_{i}$ given in [15]; ii) the attenuation $E_{\theta}\left[10 \log _{10}\left|E / E_{0}\right|^{2}\right]$ due to the combined effects of both targets does not simplify to the superposition of the effects of each target, namely $E_{\theta}\left[10 \log _{10}\left|E_{1} / E_{0}\right|^{2}\right]$ and $E_{\theta}\left[10 \log _{10}\left|E_{2} / E_{0}\right|^{2}\right]$.

\section{Multi-target localization Methods}

\section{A. Joint-ML Estimation (JML)}

Assuming the number of targets $K$ as known ${ }^{2}$, we write the model (1) for the overall set of observations as:

$$
\mathbf{s}=\Delta \mathbf{h}(\mathbf{x})+\mathbf{h}_{0}+\mathbf{w}(\mathbf{x})
$$

where $\quad \mathbf{h}_{0}=\left[h_{0,1} \cdots h_{0, L}\right]^{\mathrm{T}}$ and $\Delta \mathbf{h}(\mathbf{x})=$ $\left[\Delta h_{1}(\mathbf{x}) \cdots \Delta h_{L}(\mathbf{x})\right]^{\mathrm{T}}$ collect the empty-link mean reference and the multi-target induced deviations for all links, $\mathbf{w}(\mathbf{x})=\left[w_{1,1}(\mathbf{x}) \cdots w_{1, L}(\mathbf{x})\right]^{\mathrm{T}} \sim \mathcal{N}(\mathbf{0}, \mathbf{Q}(\mathbf{x}))$ the corresponding shadowing terms with covariance $\mathbf{Q}(\mathbf{x})=\operatorname{diag}\left(\sigma_{1,1}^{2}(\mathbf{x}), \ldots, \sigma_{1, L}^{2}(\mathbf{x})\right)$. The RSS perturbation is set to $\Delta h_{\ell}(\mathbf{x})=0$ and $\Delta \sigma_{\ell}(\mathbf{x})=0$ for $\mathbf{x}$ such that $K_{\ell}(\mathbf{x})=0$. The multi-target ML estimate is calculated as $\hat{\mathbf{x}}=\arg \max _{\mathbf{x} \in \mathcal{X}^{K}} \Lambda(\mathbf{s} \mid \mathbf{x})$ where the log-likelihood function is

$$
\Lambda(\mathbf{s} \mid \mathbf{x})=-\ln |\mathbf{Q}(\mathbf{x})|-\left\|\mathbf{s}-\Delta \mathbf{h}(\mathbf{x})-\mathbf{h}_{0}\right\|_{\mathbf{Q}(\mathbf{x})^{-1}}^{2},
$$

$|\cdot|$ denoting the determinant and $\|\mathbf{a}\|_{\mathbf{C}}^{2}=\mathbf{a}^{\mathrm{T}} \mathbf{C a}$ the weighted squared norm of a vector a weighted by the matrix $\mathbf{C}$.

\section{B. Successive Cancellation ( $S C$ )}

Successive cancellation is an iterative algorithm that localizes one target at a time. Assuming the model (5) with $K=1$ target in the monitored area, the algorithm estimates the position of a first target, $\mathbf{x}_{1}$, using the ML approach with single-target perturbation maps:

$$
\hat{\mathbf{x}}_{1}=\arg \max _{\mathbf{x}_{1} \in \mathcal{X}} \Lambda\left(\mathbf{s} \mid \mathbf{x}=\mathbf{x}_{1}\right) .
$$

\footnotetext{
${ }^{2}$ Extension to joint detection and localization of $K$ targets can be performed by extending the search space $\mathcal{X}$ for each target with a state denoting the target absence, following the approach discussed in [6] for $K=1$.
} 
The contribution of the first target is then taken into account for the estimation of the second target, as:

$$
\hat{\mathbf{x}}_{2}=\arg \max _{\mathbf{x}_{2} \in \mathcal{X}} \Lambda\left(\mathbf{s} \mid \mathbf{x}=\left[\hat{\mathbf{x}}_{1}^{\mathrm{T}}, \mathbf{x}_{2}^{\mathrm{T}}\right]^{\mathrm{T}}\right),
$$

with likelihood evaluated assuming as perturbation maps $\Delta h_{\ell}\left(\hat{\mathbf{x}}_{1}, \mathbf{x}_{2}\right)=\Delta h_{\ell}\left(\hat{\mathbf{x}}_{1}\right)+\Delta h_{\ell}\left(\mathbf{x}_{2} \mid \hat{\mathbf{x}}_{1}\right)$ and $\Delta \sigma_{\ell}^{2}\left(\hat{\mathbf{x}}_{1}, \mathbf{x}_{2}\right)=$ $\Delta \sigma_{\ell}^{2}\left(\hat{\mathbf{x}}_{1}\right)+\Delta \sigma_{\ell}^{2}\left(\mathbf{x}_{2} \mid \hat{\mathbf{x}}_{1}\right)$, where $\Delta h_{\ell}\left(\mathbf{x}_{2} \mid \hat{\mathbf{x}}_{1}\right)$ and $\Delta \sigma_{\ell}^{2}\left(\mathbf{x}_{2} \mid \hat{\mathbf{x}}_{1}\right)$ denote the contributions of the second target when the first one is located in $\hat{\mathbf{x}}_{1}$, computed using the diffraction-based model. The procedure is then repeated till the $K$ th user.

\section{Radio Tomographic Imaging (RTI)}

We propose a new tomographic imaging approach which jointly accounts for the target impact on RSS average and fluctuation. As originally proposed in [4], the DFL is formulated as the estimation of a motion image of the area $\mathcal{X}$, capturing any variation with respect to the empty-space scenario. Assuming the area $\mathcal{X}$ divided into $M$ voxels centered around the grid locations $\left\{\mathbf{b}_{m}\right\}_{m=1}^{M}$, the image to be estimated is $\mathbf{v}=\left[v_{1} \cdots v_{M}\right]^{\mathrm{T}}$ where $v_{m}=0$ or $v_{m}=1$ indicates whether a moving target is observed or not in voxel $\mathrm{m}$. For sparse motion, the RSS can be approximated as the sum of the contributions generated by all the occupied voxels:

$$
s_{\ell}=\sum_{m=1}^{M} \Delta h_{\ell, m} v_{m}+h_{0, \ell}+w_{\ell},
$$

where $\Delta h_{\ell, m}=\Delta h_{\ell}\left(\mathbf{b}_{m}\right)$ is the attenuation contribution due to a target in voxel $m$, i.e. for $\mathbf{x}=\mathbf{b}_{m}$. The corresponding power fluctuation is $w_{\ell} \sim \mathcal{N}\left(0, \sigma_{\ell}^{2}(\mathbf{v})\right)$ with $\sigma_{\ell}(\mathbf{v})=\sigma_{0, \ell}+$ $\Delta \sigma_{\ell}$ and $\Delta \sigma_{\ell}^{2}=\sum_{m=1}^{M} v_{m} \Delta \sigma_{\ell, m}^{2}$ where $\Delta \sigma_{\ell, m}=\Delta \sigma_{\ell}\left(\mathbf{b}_{m}\right)$ is the fluctuation contribution due to a target located in voxel $m$, i.e. for $\mathbf{x}=\mathbf{b}_{m}$. Considering all links, we get

$$
\mathbf{s}=\Delta \mathbf{H} \cdot \mathbf{v}+\mathbf{h}_{0}+\mathbf{w},
$$

with $\Delta \mathbf{H}=\left[\Delta h_{\ell, m}\right]$ collecting the $L \times M$ perturbations for all links/voxels, and $\mathbf{w} \sim \mathcal{N}(\mathbf{0}, \mathbf{Q}(\mathbf{v}))$ having covariance $\mathbf{Q}(\mathbf{v})=\operatorname{diag}\left(\sigma_{1}^{2}(\mathbf{v}), \ldots \sigma_{L}^{2}(\mathbf{v})\right)$ depending on the target locations. ML radio-tomographic imaging (RTI-ML) is obtained as $\hat{\mathbf{v}}=\arg \max \Lambda(\mathbf{s} \mid \mathbf{v})$ with $\Lambda(\mathbf{s} \mid \mathbf{v})=-\ln |\mathbf{Q}(\mathbf{v})|-|| \mathbf{s}-\Delta \mathbf{H v}-$ $\mathbf{h}_{0} \|_{\mathbf{Q}(\mathbf{v})^{-1}}^{2}$. Close-form least squares estimation (RTI-LS) can also be obtained as in [4], using attenuation information only: $\hat{\mathbf{v}}=\Delta \mathbf{H}^{\dagger}\left(\mathbf{s}-\mathbf{h}_{0}\right)$. Target positions are then estimated as the $K$ voxels associated with the maximum values in $\hat{\mathbf{v}}$.

\section{EXPERIMENTAL RESULTS}

Experimental validation has been performed in the seminar room of DEIB, Politecnico di Milano, using $N=18$ nodes uniformly deployed along the perimeter of a $4 \times 5 \mathrm{~m}$ area, spaced apart by $1 \mathrm{~m}$ and at $0.7 \mathrm{~m}$ of height (see Fig. 4). Each node features an NXP JN5148 single-chip wireless microcontroller, that enables applications within the $2.4 \mathrm{GHz}$ band according to the IEEE 802.15.4 standard. The RSS dynamic range is $75 \mathrm{~dB}$ with a minimum sensitivity of -95 $\mathrm{dBm}$ while the transmit power is $0 \mathrm{dBm}$. For all nodes, we employed omnidirectional, vertically polarized antennas with gain $2 \mathrm{dBi}$. The protocol consists of a modified MAC sub-layer

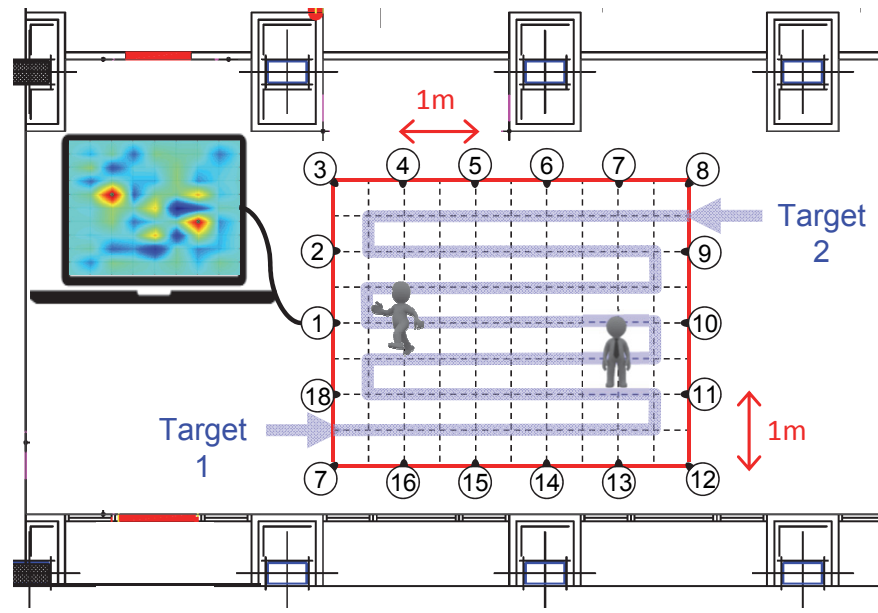

Fig. 4. Testing scenario for double-target localization: trajectory covered by the two targets and imaging example.

[7], defined on top of the beacon-enabled mode of the standard IEEE 802.15.4. A network coordinator creates a super-frame structure of duration $60 \mathrm{~ms}$ and transmits a periodic beacon frame at the beginning of each super-frame. The beacon frame allows every RF node to synchronize RSS acquisition, so that the RSSs are synchronously sampled at $60 \mathrm{~ms}$ over all links. Node 1 acts as sink, collecting data from all nodes and forwarding the information to the processing unit for imaging.

The testing layout for double-target localization is in Fig. 4. An imaging example obtained by the RTI-LS method is also superimposed, with estimated locations denoted as black crosses and real positions as green circles. The two targets follow the same trajectory but in opposite directions: target \#1 enters from the bottom-left corner, target \#2 by the top-right one. A regular grid is used for fingerprinting/analytical map evaluation, with grid points spaced by $0.5 \mathrm{~m}$ along both axes. Single-target fingerprints of RSS mean and standard deviation are computed as in [6] averaging the RSS samples over $20 \mathrm{~s}$.

In order to validate the multi-target diffraction model, the RSS perturbation is measured on link 6-14 for all possible configurations of the two targets along the grid points of the LOS path. In Fig. 5, the perturbation predicted by the diffraction model, in terms of average attenuation $\Delta h(\mathbf{x})$, is compared to the measured one, as well as to the simplified additive model based on single-target fingerprints. The diffraction-based model has been obtained by simulating two targets with dimensions $a_{y u}=b_{y u}=41 \mathrm{~cm}, a_{y v}=b_{y v}=12$ $\mathrm{cm}$, and $a_{z}=b_{z}=150 \mathrm{~cm}$. For each spatial arrangement of the two targets, the RSS moments have been evaluated by averaging over 10000 realizations of azimuth $\theta \sim \mathcal{U}(-\pi, \pi)$ and location uniformly distributed within a bin of $0.2 \times 0.2 \mathrm{~m}$ centered around the nominal grid point. Relevant multipath effects are observed in the considered indoor environment, especially due to metal foils covering the pillars along the upper and lower sides of the room (see Fig. 4). These effects are ignored by the diffraction model, while they are accounted for in fingerprinting maps as concerns the single target impact (not the interaction between the two targets). The comparison in Fig. 5 shows that the diffraction-based model better 

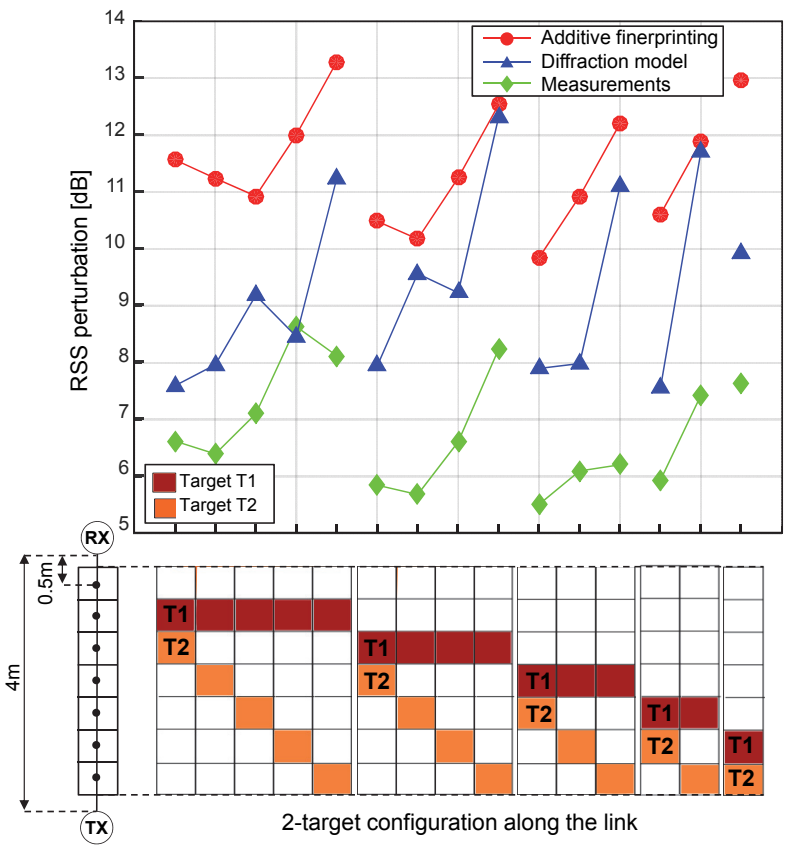

Fig. 5. Average RSS perturbation, $\Delta h(\mathbf{x})$, induced on link 6-14 by two people moving along the LOS path. The perturbation predicted by the additive model based on fingerprinting and by the diffraction model is compared to the real measurements, for the double-target configurations $\mathbf{x}$ on the bottom.

approximates the double-target shadowing with respect to the simplified additive approach, despite the mismodeling due to multipath that is not considered in the diffraction analysis (extension to estimate and compensate multipath effects is a future development).

The accuracy of multi-target localization is assessed in Fig. 6, for the algorithms JML, SC, RTI-LS and RTI-M, for known $K$. Each location fix is obtained using RSS observations collected over $4 \mathrm{~s}$. The accuracy is evaluated in terms of root mean square error (RMSE) by averaging the performances over 9 localization outcomes and over the two targets. The figure shows the RMSE vs. the trajectory steps. The best performance is provided by JML and RTI-ML, while RTI-LS performs worse as it does not exploit variance information and for SC the accuracy is limited by the single-target assumption. Note that all algorithms here perform snapshot estimates, but they can also be combined with Bayesian tracking to better handle ambiguities due to multitarget and multipath. Algorithms using analytical RSS modeling have lower accuracy compared to fingerprinting, as they trade accuracy with an easier calibration that does not require time-consuming measurement campaigns.

\section{CONCLUSiOnS}

In this paper we investigated the problem of passive localization of two moving targets in the area covered by a wireless mesh network. A diffraction-based model was proposed to describe the fading induced by the targets on each link of the network. Different estimation algorithms have been developed to infer the target locations exploiting both RSS average and variance information. Preliminary results, obtained by an experimental indoor campaign, show an average localization accuracy in the range of $0.5-1 \mathrm{~m}$.

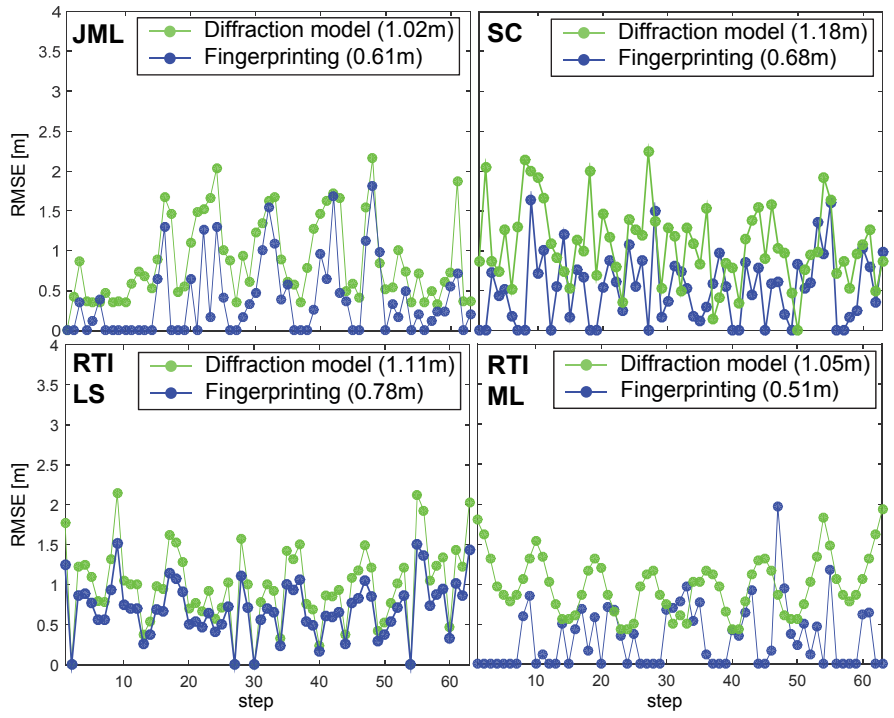

Fig. 6. Location accuracy along the trajectory for the four localization algorithms, using both diffraction-based and fingerprinting perturbation maps. The RMSE averaged over the whole trajectory is indicated on the top-right.

\section{REFERENCES}

[1] S. Savazzi, S. Sigg, M. Nicoli, V. Rampa, S. Kianoush, U. Spagnolini, "Device-free radio vision for assisted living," IEEE Signal Processing Magazine, vol. 33, n. 2, pp. 45-58, Mar. 2016.

[2] M. Youssef, M. Mah, A. Agrawala, "Challenges: device-free passive localization for wireless environments," ACM MobiCom '07, Montreal, Quebec, Canada, Sep. 9-14 2007, pp. 222-229.

[3] K. Woyach, D. Puccinelli, M. Haenggi, "Sensorless sensing in wireless networks: implementation and measurements," IEEE WiOpt '06, Boston, MA, USA, Apr. 3-7, pp.1-8.

[4] J. Wilson, N. Patwari, "Radio tomographic imaging with wireless networks," IEEE Tran. Mob. Comput., vol. 9, n. 5, 621-632, May 2010.

[5] S. Sigg, M. Scholz, S. Shi, Y. Ji, M. Beigl, "RF-sensing of activities from non-cooperative subjects in device-free recognition systems using ambient and local signals," IEEE Tran. Mob. Comput., vol. 13, n. 4, pp. 907-920, Apr. 2014.

[6] S. Savazzi, M. Nicoli, F. Carminati, M. Riva, "A Bayesian approach to Device-Free Localization: modelling and experimental assessment," IEEE J. Sel. Topics in Signal Proc., vol. 8, n. 1, pp. 16-29, Feb. 2014.

[7] S. Savazzi, V. Rampa, F. Vicentini, M. Giussani, "Device-free human sensing and localization in collaborative human-robot workspaces: a case study,” IEEE Sensors Journal, vol. 16, n. 5, pp. 1253-1264, Mar. 2016.

[8] K. Witrisal, et al., "High-accuracy localization for assisted living: 5G systems will turn multipath channels from foe to friend," IEEE Signal Processing Magazine, vol. 33, n. 2, pp. 59-70, Mar. 2016.

[9] S. Nannuru, Y. Li, M. Coates, M., B. Yang, "Multi-target devicefree tracking using radio frequency tomography," IEEE ISSNIP 2011, Adelaide, Australia, 6-9 Dec. 2011, pp. 508-513.

[10] M. Bocca, O. Kaltiokallio, N. Patwari, S. Venkatasubramanian, "Multiple target tracking with RF sensor networks," IEEE Tran. Mob. Comput., vol. 13, n. 3, pp. 1787-1800, Aug. 2014.

[11] M. Nakatsuka, H. Iwatani, J. Katto, "A study on passive crowd density estimation using wireless sensors," ICMU '08, Tokyo, June 11-13, 2008

[12] C. Xu, B. Firner, R. S. Moore,Y. Zhang ,W. Trappe, R. Howard, F. Zhang, N. An, "SCPL: indoor device-free multi-subject counting and localization using radio signal strength," ACM/IEEE IPSN 2013, 8-11 Apr. 2013, Philadelphia, PA, USA, pp. 79-90.

[13] B.R. Hamilton, M. Xiaoli, R.J. Baxley, S.M. Matechik, "Propagation modeling for radio frequency tomography in wireless networks," IEEE J. Sel. Topics in Signal Proc., vol. 8, n. 1, pp. 55-65, Feb. 2014.

[14] Z. Wang, H. Liu, S. Xu, X. Bu, J. An, "A diffraction measurement model and particle filter tracking method for RSS-based DFL," IEEE J. Sel. Topics in Signal Proc., vol. 33, n. 11, pp. 2391-2403, Nov. 2015.

[15] V. Rampa, S. Savazzi, M. Nicoli, M. D’Amico, "Physical modeling and performance bounds for device-free localization systems," IEEE Signal Proc. Letters, vol. 22, n. 11, pp. 1864-1868, Nov. 2015. 